\title{
Estudo de atividade antimicrobiana de 3,4-(metilenodióxi)-acetofenona
}

Study of the antimicrobial activity of 3,4-(methylenodioxy)-acetophenone

\author{
H. Diniz Neto ${ }^{1 *}$; J. R. Nóbrega1; G. D. Duarte ${ }^{2}$; F. V. P. Borges ${ }^{1}$; J. M. Barbosa \\ Filho $^{1}$; L. C. Rodrigues ${ }^{2}$; A. A. de Oliveira Filho ${ }^{3}$; E. O. Lima ${ }^{1}$ \\ ${ }^{1}$ Universidade Federal da Paraíba, Departamento de Ciências Farmacêuticas, Centro de Ciências da Saúde, Castelo \\ Branco III, 58059-900, João Pessoa- PB, Brasil. \\ ${ }^{2}$ Universidade Federal da Paraíba, Centro de Biotecnologia, Castelo Branco III, 58051-900, João Pessoa- PB, Brasil. \\ ${ }^{3}$ Centro de Saúde e Tecnologia Rural, Universidade Federal de Campina Grande, Jatobá, 58700-970, Patos - PB, \\ Brasil. \\ *hermes.dn@hotmail.com
}

(Recebido em 01 de agosto de 2019; aceito em 29 de janeiro de 2020)

A crescente incidência de infecções por bactérias e fungos têm preocupado diversos órgãos de saúde ao redor do mundo. Entre as infecções mais prevalentes estão as bacterianas, causadas por Staphylococcus aureus e Pseudomonas aeruginosa, e infecções fúngicas como candidíase e aspergilose. A farmacoterapia utilizada nos casos de infecção normalmente apresenta toxicidade indesejada e hoje em dia já começa a falhar ao não obter êxito diante de cepas resistentes. Esta realidade gera a necessidade crítica de desenvolvimento de novas estratégias para o combate destas infecções de elevadas taxas de mortalidade. A 3,4-(metilenodióxi)acetofenona (EPHO) é um produto químico da classe das acetofenonas, facilmente adquirido comercialmente e amplamente utilizado em reações de síntese química. Contudo, ainda não é apropriadamente explorada quanto ao seu potencial antimicrobiano. Para avaliar o perfil antimicrobiano da substância, a concentração inibitória mínima (CIM) foi determinada contra cepas bacterianas e fúngicas envolvendo tanto leveduras quanto fungos filamentosos. Foi observado que, apesar do produto se mostrar inerte contra bactérias, ele apresentou atividade inibitória contra algumas cepas de fungos leveduriformes e filamentosos com CIM respectivas de 1024 e $512 \mu \mathrm{g} / \mathrm{mL}$.

Palavras-chave: Aspergilose, candidíase, microdiluição.

The growing incidence of bacterial and fungal infections have worried several health organizations around the world. Among the most prevalent infections are the bacterial, caused by Staphylococcus aureus and Pseudomonas aeruginosa, and fungal infections such as candidiasis and aspergillosis. The pharmacotherapy used in these infection cases usually presents undesired toxicity and is currently beginning to fail due to resistant strains. This reality brings the critical need to develop new strategies to combat these infections with high mortality rates. 3,4-(methylenedioxy)-acetophenone (EPHO) is a chemical substance of the class of acetophenones, easily available commercially and widely used in chemical synthesis reactions. However, it is still not properly explored towards its antimicrobial potential. To assess the antimicrobial profile of the substance, the minimum inhibitory concentration (MIC) was determined against bacterial and fungal strains involving both yeast and filamentous fungi. It was observed that, although the product was ineffective against bacteria, it showed inhibitory activity against some strains of yeast and filamentous fungi with respective MICs of 1024 and $512 \mu \mathrm{g} / \mathrm{mL}$.

Keywords: Aspergilosis, candidiasis, microdilution.

\section{INTRODUÇÃO}

A nível mundial, o número de infecções bacterianas e fúngicas têm aumentado, trazendo como consequências altas taxas de mortalidade e alto custo associado, representando um grande desafio para os órgãos de saúde pública de diversos países [1].

Mundialmente, as bactérias são responsáveis pela maior parte das infecções humanas, aparecendo também como complicação secundária mais frequente em pacientes hospitalizados. Estas infecções são responsáveis por um vasto número de mortes com crescente aparecimento de cepas multi-resistentes, dentre as quais destacam-se espécies como Staphylococcus aureus e Pseudomonas aeruginosa [2-4]. 
Um dos tipos de infecções fúngicas mais importantes são os de candidíase, especialmente os quadros disseminados também chamados de candidemia. Estas infecções provocadas por leveduras do gênero Candida possuem elevada morbimortalidade e difícil tratamento, sobretudo quando se considera o aparecimento de linhagens resistentes [5-7].

Outra infecção fúngica de acentuada gravidade é a aspergilose, especialmente em pacientes imunossuprimidos ou hospitalizados por longos períodos, onde pode atingir taxas de mortalidade de até $80 \%[8,9]$.

A farmacoterapia utilizada para as infecções microbianas corre o risco de se tornar obsoleta devido ao aparecimento de espécies resistentes e do custo e toxicidade elevados associados a este tipo de medicamentos, criando a necessidade de se obter novos candidatos a agentes antibacterianos e antifúngicos [10].

Facilmente obtido comercialmente, a substância química 3,4-(metilenodioxi)-acetofenona (EPHO) (Figura 1) também pode ser encontrada na composição de produtos naturais como o própolis [11] e é largamente utilizada em diversos processos de síntese orgânica de produtos com as mais variadas finalidades, incluindo a obtenção de substâncias antimicrobianas [12-16].

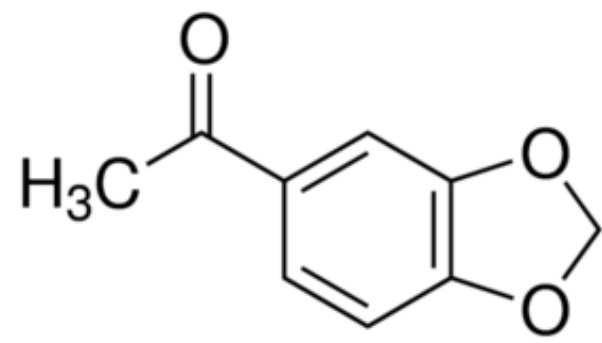

Figura 1: Estrutura química da 3,4-(metilenodioxi)-acetofenona (EPHO).

Já foi demonstrado que alguns compostos desta classe de produtos sintéticos possuem atividade antimicrobiana.

Anteriormente, Pandeya et al. (1999) [17] avaliaram a atividade antimicrobiana de derivados sintéticos obtidos a partir da 4-cloro-acetofenona, observando efeitos inibitórios moderados contra uma gama de diferentes patógenos. Eles atribuíram diferenças entre as concentrações inibitórias a particularidades estruturais entre as moléculas derivadas, tais como a presença de substituintes halogênios, além de sugerir que um possível mecanismo de ação seria através da inibição da síntese de DNA.

Outros compostos da classe das acetofenonas já demonstraram excelente efeito antifúngico contra fungos dermatófitos, com concentrações inibitórias até 16 vezes menores do que as de antifúngicos-padrão como a anfotericina $\mathrm{B}$, apontando estratégias de modificação molecular como quaternização para aprimorar o efeito biológico [18]. Ma et al. (2013) [19], apresentaram um possível grupo farmacofórico, indicando que a ocorrência simultânea de um substituinte metahidroxila fenólica e um substituinte para-alquila lipofílico beneficiavam a atividade antifúngica das moléculas derivadas das acetofenonas.

Contudo, ao se buscar na literatura, vê-se que pouco foi estudado sobre o potencial antimicrobiano utilizando a 3,4-(metilenodioxi)-acetofenona como produto-teste principal contra microrganismos de importância para a saúde pública, onde vários trabalhos avaliaram apenas o potencial de outros análogos e produtos obtidos a partir da EPHO após outras rotas sintéticas. Uma vez que a busca por novos agentes antimicrobianos é fortemente incentivada, buscou-se observar o potencial antifúngico e antibacteriano de uma substância amplamente utilizada nos processos de síntese, porém ainda pouco estudada em relação ao seu potencial biológico.

\section{MATERIAL E MÉTODOS}

O produto-teste 3,4-(metilenodioxi)-acetofenona (EPHO) e os antimicrobianos utilizados como controle, cloranfenicol, nistatina e fluconazol, foram obtidos na Sigma-Aldrich (São Paulo, SP, Brasil), o dimetilsulfóxido (DMSO) e Tween 80 foram comprados na Labsynth Ltda. (Diadema, SP, Brasil). Os produtos foram solubilizados em DMSO a uma proporção de 5\%, Tween 80 a $2 \%$ 
e completando o volume final com água destilada esterilizada, de forma a se obter uma emulsão cuja concentração inicial nos ensaios será de $1024 \mu \mathrm{g} / \mathrm{mL}$ para o EPHO, enquanto a concentração estabelecida para os antimicrobianos será de $100 \mu \mathrm{g} / \mathrm{mL}$. As misturas foram agitadas durante 3 minutos em um aparelho Vortex (Fanem ${ }^{\circledR}$ Ltda., Guarulhos, SP, Brasil). As emulsões dos produtos utilizadas nos ensaios antifúngicos foram preparadas no momento da execução dos testes.

Os meios de cultura utilizados foram: Brain Heart Infusion (BHI) e Agar Sabouraud Dextrose (ASD) (Difco Laboratories Ltd, USA, France) para manutenção das cepas, e caldo Brain Heart Infusion (BHI) e meio RPMI 1640 com L-glutamina e sem bicarbonato para os ensaios de atividade biológica contra bactérias e fungos, respectivamente (Difco Laboratories Ltd, USA, France e INLAB, São Paulo, Brasil). Os meios foram preparados de acordo com as instruções dos fabricantes. Os meios foram solubilizados em água destilada e esterilizados em autoclave a $121{ }^{\circ} \mathrm{C}$, 1,0 atm durante $15 \mathrm{~min}$.

Para a realização dos ensaios, foram escolhidos microrganismos variando entre bactérias, fungos leveduriformes e fungos filamentosos: Staphylococcus aureus ATCC-13150, Staphylococcus aureus ATCC-6538, Pseudomonas aeruginosa ATCC-25853, Pseudomonas aeruginosa ATCC9027, Candida albicans ATCC-60193, Candida tropicalis ATCC-13803, Candida parapsilosis ATCC-22019, Candida glabrata LM-116, Aspergillus fumigatus ATCC-40640 e Aspergillus flavus LM-714. Os microrganismos pertencem a MICOTECA do Laboratório de Micologia, Departamento de Ciências Farmacêuticas (DCF), Centro de Ciências da Saúde (CCS) da Universidade Federal da Paraíba (UFPB). As cepas bacterianas e fúngicas foram mantidas respectivamente em $\mathrm{BHI}$ e $\mathrm{ASD}$ à temperatura de $4^{\circ} \mathrm{C}$. Foram utilizados para os ensaios, repiques de 24-48 horas em BHI e ASD, respectivamente, para bactérias e fungos, incubados a $35 \pm 2^{\circ} \mathrm{C}$; e repiques de 7-14 dias em ASD para fungos filamentosos, incubados a $28 \pm 2{ }^{\circ} \mathrm{C}$. Para preparação do inóculo, as colônias obtidas de culturas das cepas bacterianas e fúngicas foram suspensas em solução fisiológica estéril a $0,9 \%$ e ajustadas de acordo com o tubo 0,5 da escala padrão de McFarland para obtenção de $10^{6}$ e $10^{8}$ Unidades Formadoras de Colônia por mL (UFC/mL) para fungos e bactérias, respectivamente [20-25].

A CIM foi determinada pelo método da microdiluição [26]. Inicialmente, foram distribuídos 100 $\mu \mathrm{L}$ de caldo sendo RPMI para fungos e BHI para bactérias, duplamente concentrado nos orifícios das placas de microdiluição para cultura de células (TPP/Suíça) contendo 96 poços com fundo em "U". Em seguida, $100 \mu \mathrm{L}$ da emulsão dos produtos preparados foram dispensados nas cavidades da primeira linha da placa e por meio de uma diluição seriada a uma razão de dois, foi obtida concentrações de $1024 \mu \mathrm{g} / \mathrm{mL}$ até $0,5 \mu \mathrm{g} / \mathrm{mL}$. Por fim, foi adicionado $10 \mu \mathrm{L}$ das suspensões das cepas bacterianas e fúngicas nas cavidades.

Paralelamente, foram realizados os controles de viabilidade de microrganismos (BHI + bactérias e RPMI + leveduras ou fungos filamentosos), esterilidade dos meios de cultura (RPMI/BHI) e controles negativos utilizando antimicrobianos padronizados, cloranfenicol $(100 \mu \mathrm{g} / \mathrm{mL})$ para bactérias, nistatina $(100 \mu \mathrm{g} / \mathrm{mL})$ para leveduras e fluconazol $(100 \mu \mathrm{g} / \mathrm{mL})$ para fungos filamentosos. Para verificar a ausência de interferência nos resultados pelos solventes utilizados na preparação da emulsão (DMSO e Tween 80), foi feito um controle no qual foram colocados nas cavidades da microplaca o meio RPMI 1640, DMSO a 5\%, Tween 80 a $2 \%$ e o inóculo fúngico. As placas preparadas foram assepticamente fechadas e submetidas à incubação numa temperatura de $35 \pm$ $2^{\circ} \mathrm{C}$ por $24-48$ horas para os ensaios com bactérias e leveduras, e $28 \pm 2^{\circ} \mathrm{C}$ por 7 dias para fungos filamentosos.

A CIM foi definida como a menor concentração do produto que inibiu o crescimento visível do microrganismo no poço da microplaca. Os ensaios foram realizados em triplicata e os resultados foram expressos como a média aritmética da CIM obtidas nos experimentos.

\section{RESULTADOS E DISCUSSÃO}

Na Tabela 1 estão registrados os resultados obtidos no ensaio de avaliação da atividade antibacteriana e antifúngica dos produtos. Em relação aos controles efetuados, não houve crescimento microbiano no controle de esterilidade do caldo enquanto os inóculos apresentaram crescimento abundante no controle de viabilidade. 
Tabela 1: Concentração inibitória mínima $(C I M)(\mu \mathrm{g} / \mathrm{mL})$ do EPHO, cloranfenicol, nistatina e fluconazol contra bactérias, leveduras e fungos filamentosos.

\begin{tabular}{|c|c|c|c|c|c|c|}
\hline \multirow{2}{*}{ Microrganismos } & \multicolumn{4}{|c|}{ Substâncias } & \multirow{2}{*}{$\begin{array}{l}\text { Controle de } \\
\text { esterilidade }\end{array}$} & \multirow{2}{*}{$\begin{array}{l}\text { Controle de } \\
\text { crescimento }^{\mathrm{a}}\end{array}$} \\
\hline & EPHO & Clor & Nist & Fluc & & \\
\hline S. aureus ATCC-13150 & $\mathrm{N} / \mathrm{D}$ & 100 & N/A & N/A & - & + \\
\hline S. aureus ATCC-6538 & $\mathrm{N} / \mathrm{D}$ & 100 & N/A & N/A & - & + \\
\hline P. aeruginosa ATCC-9027 & $\mathrm{N} / \mathrm{D}$ & 100 & N/A & N/A & - & + \\
\hline P. aeruginosa ATCC-25853 & N/D & 100 & N/A & N/A & - & + \\
\hline C. albicans ATCC-60193 & 1024 & N/A & 100 & N/A & - & + \\
\hline C. tropicalis ATCC-13803 & N/D & N/A & 100 & N/A & - & + \\
\hline C. parapsilosis ATCC-22019 & 1024 & N/A & 100 & N/A & - & + \\
\hline C. glabrata LM-116 & 1024 & N/A & 100 & N/A & - & + \\
\hline A. fumigatus ATCC-40640 & 512 & N/A & N/A & 100 & - & + \\
\hline A. flavus LM-714 & N/D & N/A & N/A & 100 & - & + \\
\hline
\end{tabular}

Clor, cloranfenicol; Nist, nistatina; Fluc, fluconazol; N/D, não determinado para as concentrações utilizadas; N/A, não aplicado; -, ausência de crescimento fúngico; +, presença de crescimento fúngico; ${ }^{a}$ crescimento fúngico em RPMI-1640, DMSO (5\%) e Tween 80 (2\%), sem agente antifúngico.

Mesmo na mais alta concentração $(1024 \mu \mathrm{g} / \mathrm{mL})$, o produto EPHO não foi capaz de inibir as cepas $S$. aureus ATCC-13150, S. aureus ATCC-6538, P. aeruginosa ATCC-9027, P. aeruginosa ATCC-25853, C. tropicalis ATCC-13803 e A. flavus LM-714.

Contudo, o EPHO inibiu o crescimento microbiano das cepas C. albicans ATCC-60193, C. parapsilosis ATCC-22019 e C. glabrata LM-116 na concentração de $1024 \mu \mathrm{g} / \mathrm{mL}$, assim como também inibiu o crescimento da cepa A. fumigatus ATCC-40640 na concentração de $512 \mu \mathrm{g} / \mathrm{mL}$.

Em resumo, o produto EPHO não demonstrou atividade antibacteriana nas concentrações utilizadas, apenas atividade antifúngica contra algumas leveduras e uma espécie de fungo filamentoso, sendo eficaz contra este último microrganismo ao apresentar menor CIM.

Como não há um consenso bem estabelecido sobre a potência e natureza da atividade inibitória de novos produtos, a classificação exata sobre a eficácia da atividade inibitória demonstrada por uma substância inédita torna-se difícil. De acordo com os critérios estabelecidos por Sartoratto et al. (2004), pode-se estabelecer que o EPHO é uma substância de moderada atividade antifúngica, visto que sua CIM de $1024 \mu \mathrm{g} / \mathrm{mL}$ para as leveduras sensíveis e $512 \mu \mathrm{g} / \mathrm{mL}$ para A. fumigatus, está dentro da faixa de concentração determinante para atividade antimicrobiana moderada (entre 1500 e $600 \mu \mathrm{g} / \mathrm{mL}$ ) [27].

De ampla distribuição mundial, a candidíase tem adquirido relevância pela emergente ocorrência de cepas resistentes tanto de $C$. albicans quanto de espécies não-albicans. Além disso, mesmo as afecções superficiais comuns provocadas por este fungo podem apresentar sérios problemas ao paciente devido a exacerbação das lesões e respostas imunológicas, especialmente em quadros de co-infecção [28]. Outro agravante é o dimorfismo deste gênero fúngico, onde a alteração morfológica para a forma de pseudohifa representa uma perigosa evolução da infecção ao conferir resistência e facilitar a disseminação sistêmica, com ocorrência de danos teciduais frequentes [29].

Recentemente, tem sido notado um drástico aumento dos casos de aspergilose, especialmente em indivíduos imunocomprometidos. Já foi demonstrado que o gênero Aspergillus, principalmente A. fumigatus, possui elevada patogenicidade e virulência, possuindo fatores intrínsecos como: resistência a altas e baixas, formação de biofilme, liberação de proteases e micotoxinas, evasão das respostas imunes do hospedeiro, tolerância a estresse oxidativo e possibilidade de sobrevivência em ambientes de baixo $\mathrm{pH}$. A complexidade deste patógeno favorece a infecção e facilmente torna crítico o quadro de saúde do paciente [30].

Por se tratar de uma substância ainda com poucos estudos sob a ótica da atividade antifúngica, há uma deficiência de dados na literatura sobre a 3,4-(metilenodioxi)-acetofenona para que se possa ser feita uma comparação apropriada com os resultados do presente estudo, sobretudo ao considerar que alguns destes poucos estudos empregaram linhagens e microrganismos diferentes. 
Previamente, Sivakumar et al. (2008) [31] avaliaram o perfil antibacteriano de diversas substâncias pertencentes à classe das acetofenonas, incluindo o próprio EPHO. Como resultado, viram que este composto possuía baixíssima atividade inibitória contra os microrganismos selecionados, com uma cepa de $S$. aureus entre eles, apresentando uma CIM de valor elevado $\left(3,7 \times 10^{5} \mathrm{M}\right)$, superior ao que foi obtido no trabalho atual, o que corrobora com os atuais dados ao demonstrar que seria necessário altas concentrações desta substância para ser possível a observação de algum efeito antibacteriano.

Apesar de ainda não haver dados sobre a atividade antifúngica do EPHO, outras moléculas da classe das acetofenonas e derivados já demonstraram atividade contra diferentes fungos, incluindo C. albicans, sugerindo também que sua ação farmacológica provavelmente ocorra devido a inibição da formação da parede celular fúngica além de impedir o desenvolvimento de estruturas de virulência como pseudohifas $[19,32]$.

A síntese de compostos a partir de acetofenonas na tentativa de se obter agentes antimicrobianos continuou, como descrito por Mete et al. (2011) [33], ao obter derivados capazes de inibir o crescimento fúngico de maneira mais eficaz do que a anfotericina $\mathrm{B}$. Também foi apontado que estas substâncias exerçam sua atividade inibitória através da alquilação de grupos tiol presentes nas proteínas fúngicas e que esta atividade é influenciada pela substituição do anel fenílico pelo anel tiofênico.

Vale destacar que a acetofenona do presente trabalho manteve o grupo carbonila em semelhança ao modelo farmacofórico proposto, entretanto, não houve adição de um halogênio, o que poderia justificar a atividade em nível moderado. Contudo, é a primeira vez em que se utiliza um grupo metilenodióxi no anel principal, sendo necessária a avaliação de outros análogos mantendo este mesmo núcleo para estabelecer a sua influência na atividade antifúngica.

Através das técnicas de modificação molecular e estudos de relação estrutura química e atividade biológica, pode-se desenvolver um produto com maior eficácia bioativa e menor toxicidade partindo de um produto que já tenha demonstrado atividade antimicrobiana, mesmo que moderada. Sendo assim, ao realizar experimentos empregando diretamente a EPHO e trazer dados da literatura sobre o potencial de outras substâncias, esta pesquisa incentiva e fornece subsídios para o desenvolvimento de outros trabalhos que se proponham a melhorar o perfil antimicrobiano da 3,4-(metilenodioxi)-acetofenona, chegando cada vez mais perto de se obter um fármaco adequado para utilização na terapêutica antifúngica.

Devido a gravidade das infecções fúngicas, a escassez terapêutica, toxicidade dos medicamentos disponíveis e surgimento de cepas resistentes, novas substâncias com potencial de serem utilizadas no combate destes patógenos representam uma valiosa chance de se contornar uma realidade preocupante, garantindo melhores chances de sobrevivência para os pacientes acometidos por este tipo de infecção $[8,34,35]$.

\section{CONCLUSÃO}

Os resultados obtidos neste trabalho a respeito de uma substância cujo potencial antimicrobiano ainda não foi profundamente estudado servem como dados preliminares para outros trabalhos que possam ser realizados para se investigar a fundo a natureza bioativa deste produto. Como exemplo, pode-se citar a experimentação de novos análogos obtidos a partir da molécula e a investigação dos possíveis mecanismos de ação envolvidos na atividade antifúngica, além de considerar também a possibilidade de atuação em outras vias de ação farmacológica e de se estabelecer o perfil de toxicidade deste produto e seus derivados.

Ao mesmo tempo, este trabalho reúne dados que possam incentivar e direcionar o uso desta e de outras moléculas da classe das acetofenonas como modelos para a obtenção de novos e eficazes agentes antifúngicos, utilizando dos conhecimentos de relação estrutura-atividade para se obter o produto de melhor desempenho. Vale ainda salientar que além de estabelecer os possíveis mecanismos de ação envolvidos na atividade antifúngica e analisar as estratégias de modificação estrutural, também se faz necessário o estudo do perfil toxicológico destas substâncias para que se possa chegar a um fármaco que além de eficaz, também seja seguro para ser inserido na terapia antifúngica. 


\section{REFERÊNCIAS BIBLIOGRÁFICAS}

1. Bloom DE, Black S, Rappuoli R. Emerging infectious diseases: a proactive approach. Proc Natl Acad Sci. 2017;201701410.

2. Greenhow TL, Hung Y-Y, Herz AM, Losada E, Pantell RH. The changing epidemiology of serious bacterial infections in young infants. Pediatr Infect Dis J. 2014;33(6):595-9.

3. Lachiewicz AM, Hauck CG, Weber DJ, Cairns BA, Van Duin D. Bacterial infections after burn injuries: impact of multidrug resistance. Clin Infect Dis. 2017;65(12):2130-6.

4. Seale AC, Agarwal R. Improving management of neonatal infections. The Lancet. 14 de julho de 2018;392(10142):100-2.

5. Colombo AL, Guimarães T, Sukienik T, Pasqualotto AC, Andreotti R, Queiroz-Telles F, Nouér SA, Nucci M. Prognostic factors and historical trends in the epidemiology of candidemia in critically ill patients: an analysis of five multicenter studies sequentially conducted over a 9-year period. Intensive Care Med. 2014;40(10):1489-98.

6. Kim S-H, Yoon YK, Kim MJ, Sohn JW. Clinical impact of time to positivity for Candida species on mortality in patients with candidaemia. J Antimicrob Chemother. 2013 Dec;68(12):2890-7.

7. Xisto MIDS, Caramalho RDF, Rocha DAS, Ferreira-Pereira A, Sartori B, Barreto-Bergter E, Junqueira ML, Läss-Florl C, Lackner M. Pan-azole-resistant Candida tropicalis carrying homozygous erg11 mutations at position K143R: a new emerging superbug? J Antimicrob Chemother. 2017 Apr;72(4):988-92.

8. Lestrade PP, van der Velden WFJM, Bouwman F, Stoop FJ, Blijlevens NMA, Melchers WJG, Verweij PE, Donnelly JP. Epidemiology of invasive aspergillosis and triazole-resistant Aspergillus fumigatus in patients with haematological malignancies: a single-centre retrospective cohort study. J Antimicrob Chemother. 2018;73(5):1389-94.

9. Taccone FS, Van den Abeele A-M, Bulpa P, Misset B, Meersseman W, Cardoso T, Paiva JA, BlascoNavalpotro M, De Laere E, Dimopoulos G, Rello J, Vogelaers D, Blot SI. Epidemiology of invasive aspergillosis in critically ill patients: clinical presentation, underlying conditions, and outcomes. Crit Care. 2015;19(1):7.

10. Kirby T. Europe to boost development of new antimicrobial drugs. The Lancet. 2012 Jun;379(9833):2229-30.

11. Muñoz Orlando, Peña Raúl C., Ureta Enrique, Montenegro Gloria, Timmermann Barbara N. Propolis from Chilean Matorral Hives. Z Für Naturforschung C. 2014;56(3-4):269.

12. Giddens AC, Boshoff HIM, Franzblau SG, Barry CE, Copp BR. Antimycobacterial natural products: synthesis and preliminary biological evaluation of the oxazole-containing alkaloid texaline. Tetrahedron Lett. 2005 Oct;46(43):7355-7.

13. Marson C, Schneider S, Meys F, Wennig R. Structural elucidation of an uncommon phenylethylamine analogue in urine responsible for discordant amphetamine immunoassay results. J Anal Toxicol. 2000 Jan;24(1):17-21.

14. Ramulu BJ, Chanda T, Chowdhury S, Nandi GC, Singh MS. Organocatalyzed straightforward synthesis of highly fluorescent 3,5-disubstituted 2,6-dicyanoanilines via domino annulation of $\alpha$ enolicdithioesters with malononitrile. RSC Adv. 2013;3(16):5345-9.

15. Rostom SAF, Badr MH, Razik HAAE, Ashour HMA, Wahab AEA. Synthesis of some pyrazolines and pyrimidines derived from polymethoxy chalcones as anticancer and antimicrobial agents. Arch Pharm (Weinheim). 2011;344(9):572-87.

16. Sommerwerk S, Kern S, Heller L, Csuk R. First total synthesis of piperodione and analogs. Tetrahedron Lett. 2014 Nov;55(45):6243-4.

17. Pandeya S, Sriram D, Nath G, DeClercq E. Synthesis, antibacterial, antifungal and anti-HIV activities of Schiff and Mannich bases derived from isatin derivatives and N-[4-(4'-chlorophenyl) thiazol-2-yl] thiosemicarbazide. Eur J Pharm Sci. 1999;9(1):25-31.

18. Gul HI, Ojanen T, Vepsalainen J, Gul M, Erciyas E, Hanninen O. Antifungal activity of some mono, bis and quaternary Mannich bases derived from acetophenone. Arzneimittelforschung. 2001;51(01):725 .

19. Ma Y, Fan H, Gao Y, Li H, Zhang A, Gao J. Natural products as sources of new fungicides (i): synthesis and antifungal activity of acetophenone derivatives against phytopathogenic fungi. Chem Biol Drug Des. 2013;81(4):545-52.

20. Clinical and Laboratory Standards Institute. Reference method for broth dilution antifungal susceptibility testing of yeasts; approved standard. CLSI Doc M27-A3 Suppl S. 2008;(3).

21. Clinical and Laboratory Standards Institute. Methods for Dilution Antimicrobial Susceptibility Tests for Bacteria That Grow Aerobically; Approved Standard. CLSI Doc M07-A9. 2012;(9). 
22. Clinical and Laboratory Standards Institute. Reference method for broth dilution antifungal susceptibility testing of filamentous fungi; Approved standard. CLSI Doc M38-A2. 2008;(2).

23. CLSI Performance standards for antimicrobial susceptibility testing. Ninet Informational Suppl CLSI Doc M100-S21 Wayne PA Clin Lab Stand Inst. 2011;

24. Hadacek F, Greger H. Testing of antifungal natural products: methodologies, comparability of results and assay choice. Phytochem Anal. 2000;11(3):137-47.

25. Cleeland R, Squires E. Evaluation of new antimicrobials in vitro and in experimental animal infections. Antibiot Lab Med. 1991;3:739-87.

26. Eloff JN. A sensitive and quick microplate method to determine the minimal inhibitory concentration of plant extracts for bacteria. Planta Med. 1998;64(08):711-3.

27. Sartoratto A, Machado ALM, Delarmelina C, Figueira GM, Duarte MCT, Rehder VLG. Composition and antimicrobial activity of essential oils from aromatic plants used in Brazil. Braz J Microbiol. 2004;35(4):275-80.

28. Xu H, Sobue T, Thompson A, Xie Z, Poon K, Ricker A, Cervantes J, Diaz PI, Dongari-Bagtzoglou A. Streptococcal co-infection augments Candida pathogenicity by amplifying the mucosal inflammatory response. Cell Microbiol. 2014;16(2):214-31.

29. Mishra S, Singh S, Misra K. Restraining pathogenicity in Candida albicans by taxifolin as an inhibitor of Ras1-pka pathway. Mycopathologia. 2017;182(11-12):953-65.

30. Paulussen C, Hallsworth JE, Álvarez-Pérez S, Nierman WC, Hamill PG, Blain D, Rediers H, Lievens B. Ecology of aspergillosis: insights into the pathogenic potency of Aspergillus fumigatus and some other Aspergillus species. Microb Biotechnol. 2017;10(2):296-322.

31. Sivakumar PM, Sheshayan G, Doble M. Experimental and QSAR of acetophenones as antibacterial agents. Chem Biol Drug Des. 2008 Sep;72(4):303-13.

32. Soberón JR, Lizarraga EF, Sgariglia MA, Juárez MBC, Sampietro DA, Altabef AB, Catalán CAN, Vattuone MA. Antifungal activity of 4-hydroxy-3-(3-methyl-2-butenyl) acetophenone against Candida albicans: evidence for the antifungal mode of action. Antonie Van Leeuwenhoek. 2015 Nov;108(5):1047-57.

33. Mete E, Gul HI, Bilginer S, Algul O, Topaloglu ME, Gulluce M, Kazaz C. Synthesis and antifungal evaluation of 1-Aryl-2-dimethyl-aminomethyl-2-propen-1-one hydrochlorides. Molecules. 2011;16(6):4660-71.

34. Jensen RH, Astvad KMT, Silva LV, Sanglard D, Jørgensen R, Nielsen KF, Mathiasen EG, Doroudian G, Perlin DS, Arendrup MC. Stepwise emergence of azole, echinocandin and amphotericin B multidrug resistance in vivo in Candida albicans orchestrated by multiple genetic alterations. J Antimicrob Chemother. 2015;70(9):2551-5.

35. Motta FA, Dalla-Costa LM, Muro MD, Cardoso MN, Picharski GL, Jaeger G, Burger M. Risk factors for candidemia mortality in hospitalized children. J Pediatr (Rio J). 2017;93(2):165-71. 